\title{
Patellar Pseudoarthrosis With Intact Knee Function - A Case Report
}

\section{Sameer Panchal ${ }^{1 *}$, Sangeet Gawhale ${ }^{2}$, Nadir Z Shah ${ }^{3}$ and Sujith $\mathbf{B S}^{4}$}

${ }^{1}$ Assistant Professor, Department of Orthopaedics, Grant Medical College and Sir JJ Group of Hospitals, Byculla, Mumbai, India

${ }^{2}$ Professor and Head of the unit, Department of Orthopaedics, Grant Medical

College and Sir JJ Group of hospitals, Byculla, Mumbai, India

${ }^{3}$ Associate Professor, Department of Orthopaedics, Grant medical college and Sir JJ

group of hospitals, Byculla, Mumbai, India

${ }^{4}$ Senior Resident, Department of Orthopaedics, TNMC and B.Y.L Nair Hospital,

Mumbai, India

*Corresponding Author: Sameer Panchal, Assistant Professor, Department of Orthopaedics, Grant Medical College and Sir JJ Group of Hospitals, Byculla, Mumbai, India.
Received: January 08, 2021

Published: Janaury 28, 2021

(C) All rights are reserved by Sameer Panchal., et al.

\section{Abstract}

Case Report: 65 year old male, tailor by occupation sustained transverse fracture of patella following trauma due to fall and was referred to our centre. Open reduction and internal fixation were performed and patient was mobilized post operatively as per the routine protocol. Patient regained full movements in two months and was back to his normal job in three months. However, Implant removal was done two years later after the index surgery in view of discharging sinus. His patellar fracture has not healed with obvious fracture gap but he is able carry activities of daily living, climbing stairs with weights and has some knee pain.

Conclusion: Despite of patellar non-union of four years, patient has full active knee range of motion and no extensor lag and is able to do all heavy activities. Managing non-union of patella is an orthopedic challenge but the real question arises does all the patients with non-union patella requires some form of intervention.

Keywords: Patella; Trauma; Lever Arm

\section{Introduction}

Patella fractures constitute $1 \%$ of all skeletal fractures. It plays a vital role biomechanically by improving quadriceps efficiency and increasing the lever arm of the extensor mechanism. Intact patella is of prime importance for normal flexion-extension of knee. It forms an integral part of extensor apparatus of knee by converting tensile force into compressive force and hence decelerates knee flexion during walking, climbing steps or walking downhill. This is known as patella-femoral joint reaction force. Patella increases the lever arm of the quadriceps by displacing the linkage between quadriceps and proximal tibia away from the axis of knee rotation during flexion between 45-60 degrees. Direct or indirect injury to patella causes patella fracture with disruption of extensor apparatus leading to eccentric contraction of quadriceps.

Patella non-union is a known complication and as quoted by the literature the incidence varies between $2.7 \%$ to $12.5 \%$. There is no treatment protocol described for patella non-union. Management of patella non-union is an orthopedic challenge which relates to 
proximal displacement of bony fragment, soft tissue contractures, associated extensor lag and joint stiffness. Most of the patients require some sort of intervention considering their age and functional demand. Preserved knee range with no extensor lag is an extremely rare phenomenon in patella non-union and very few cases have reported

\section{Informed consent}

Patient has been informed that data concerning the case would be submitted for the purpose of publication and the patient agreed for the same.

\section{Case Presentation}

We report a case of 65 years old male individual who presented to us with knee pain while performing ground level activities. He had a history of fall four years back and sustained blunt trauma to right knee. He sustained a transverse fracture of patella which was managed surgically with open reduction and internal fixation by 18 gauge figure of eight tension band wiring (TBW) and cerclage. Patient was mobilized and knee range of motion was started with partial weight bearing immediately after the surgery. Patient was asymptomatic for two years with good range of motion and function, later came with discharging sinus over anterior aspect of knee. Implant removal and debridement was done to tackle infection and non-union was planned to be managed in second stage.

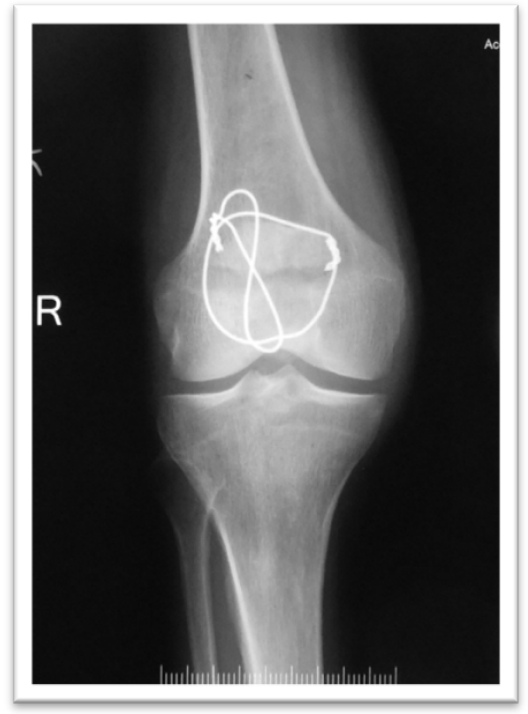

Figure 1: Post-operative radiograph showing antero-posterior view of right knee.

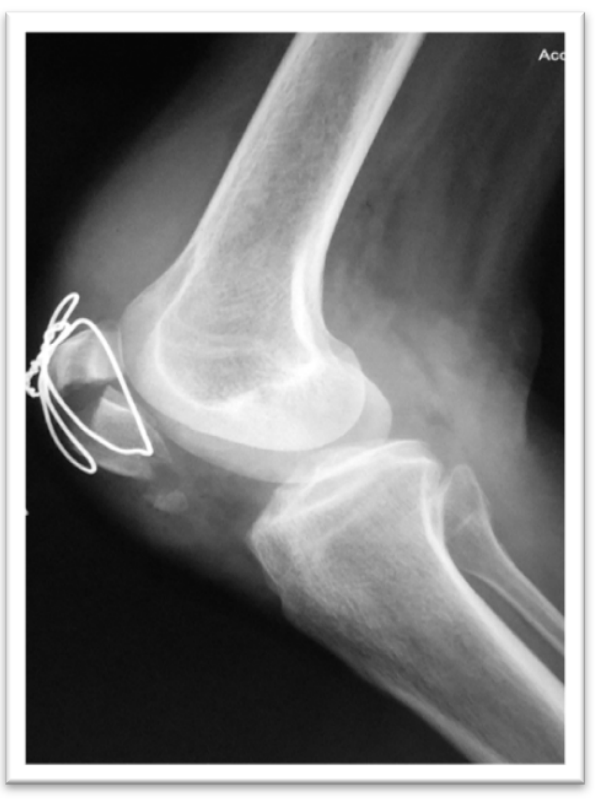

Figure 2: Post-operative radiograph showing lateral view of right knee with mal-reduced patella.

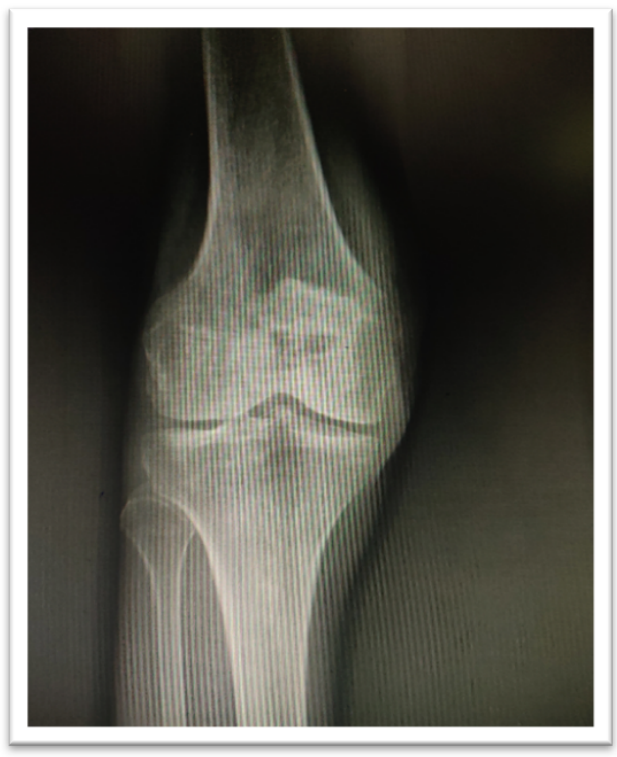

Figure 3: Antero-posterior radiograph of right knee after implant removal. 


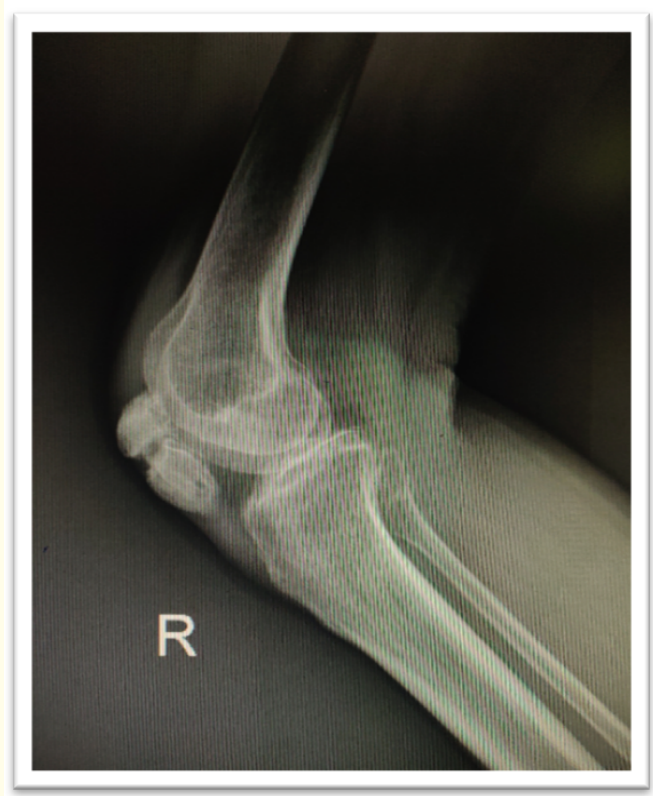

Figure 4: Lateral radiograph of right knee showing visible defect at the fracture site.

Infection was completely cured and surprisingly to our notice patient had complete knee range of motion with no extensor lag despite of radiological evidence of patella non-union. Patient is able to perform active straight leg raise test and is walking full weight bearing without assistance. The only complaint patient currently had was knee pain predominantly while performing ground level activities.

\section{Discussion}

Patella non-union is a well-known but quite rare complication of patella fractures with very low incidence varying from $1 \%$ to $12.5 \%$ [1]. Most of the research emphasizes on management of patella non-union by different methods as there is no standard protocol for treatment. The purpose of this case report is to emphasize whether every patient with patellar non-union do require some operative intervention considering age and functional demand of the patient or can be tackled conservatively.

Management of patellar non-union depends solely on patient's functional demand. A study by Garg et.al compared various surgical methods for treating patellar non-union in patients with high

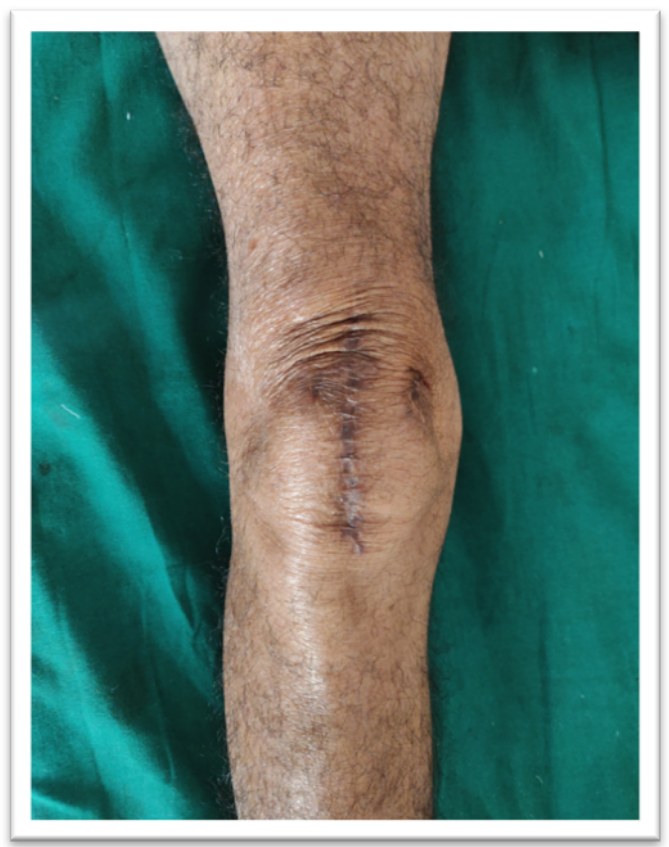

Figure 5: Skin condition of the patient after implant removal and debridement.

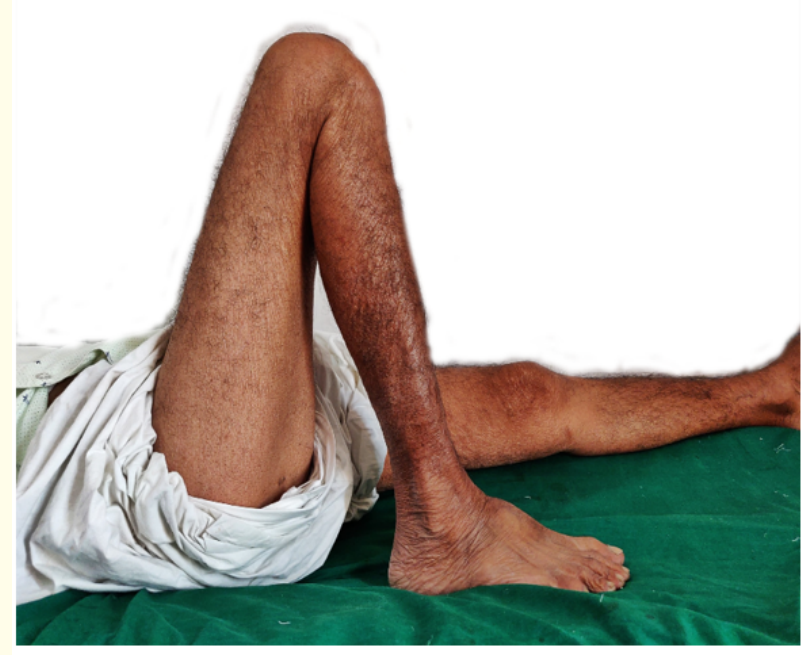

Figure 6: Image showing complete active knee flexion. 


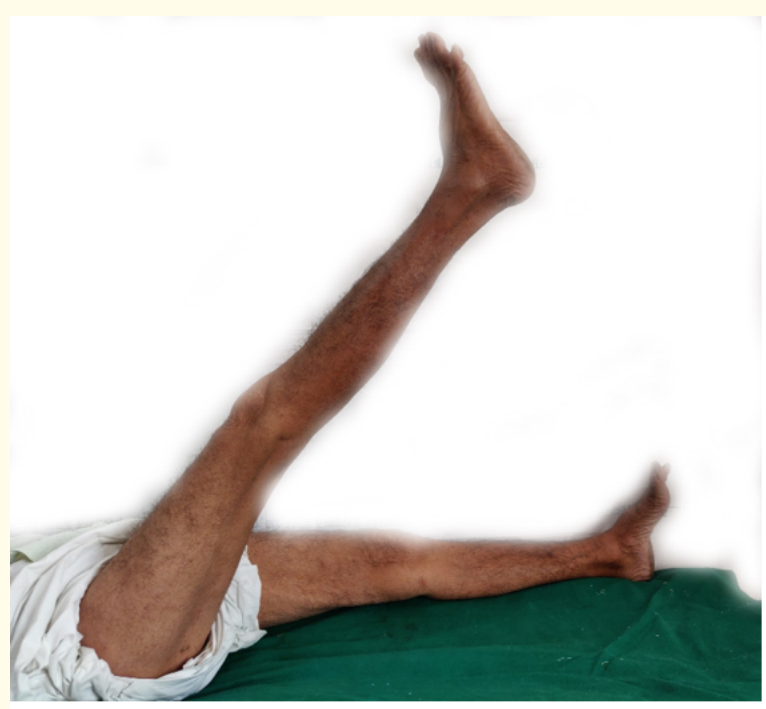

Figure 7: Image showing active straight leg raising in supine position.

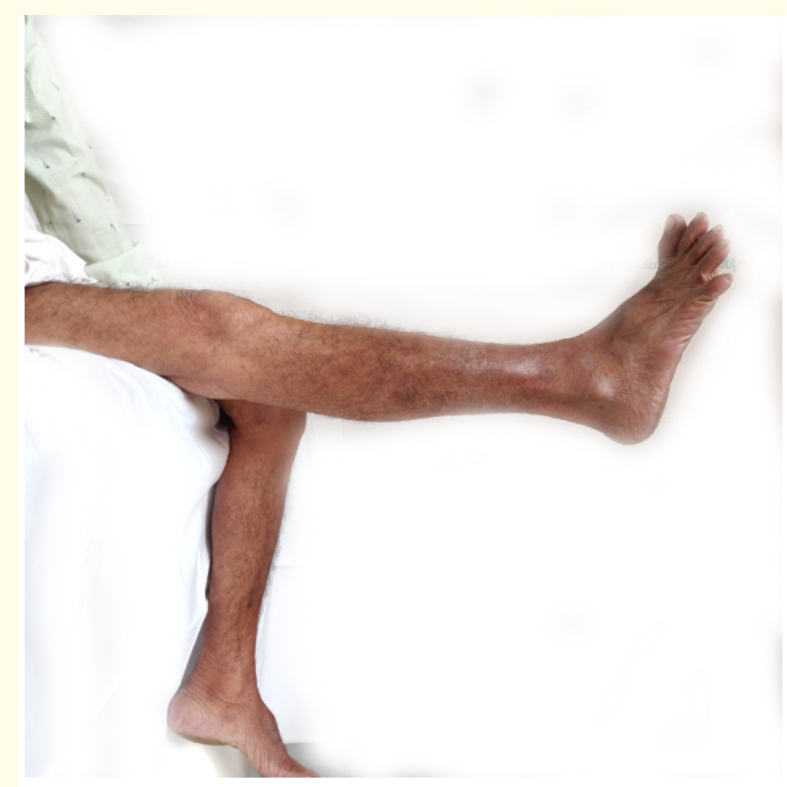

Figure 8: Active straight leg raising alongside bed.

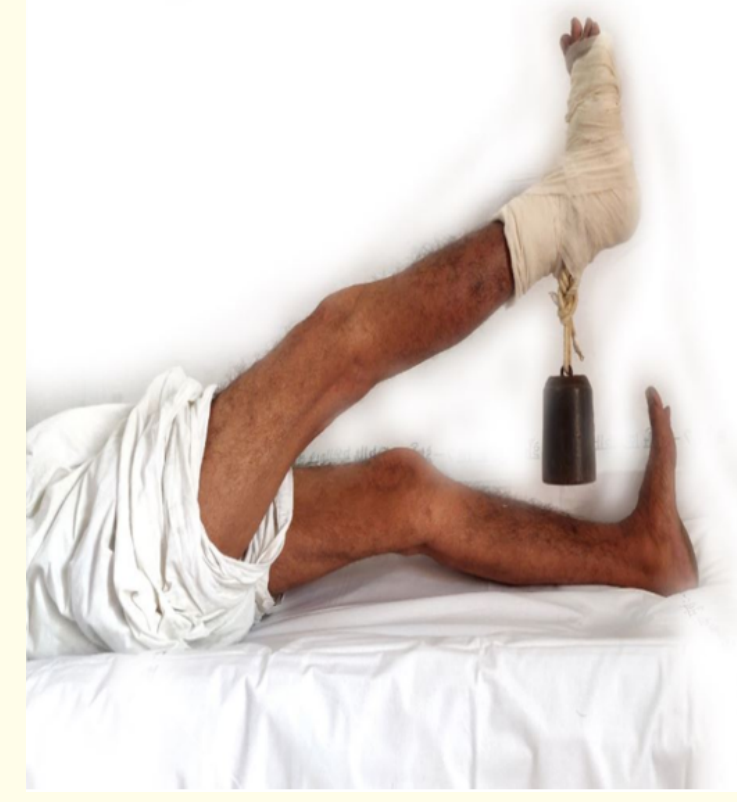

Figure 9: Active straight leg raising with ankle weights.

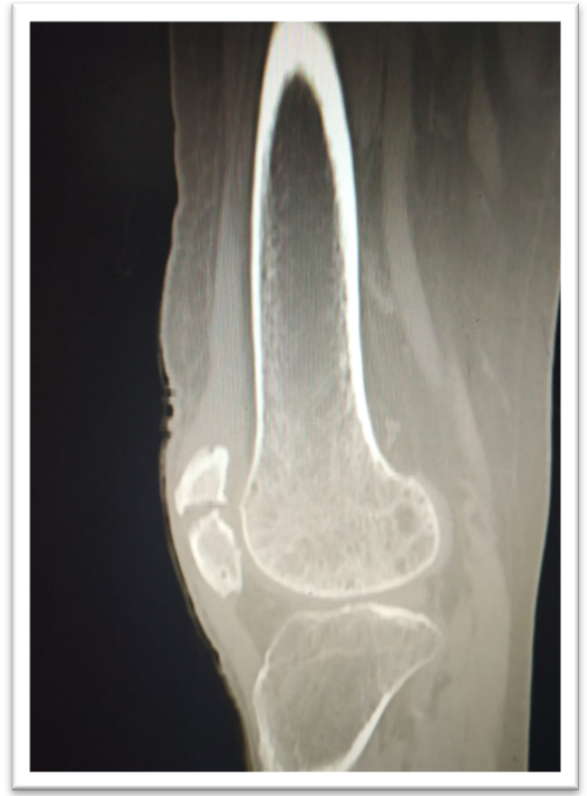

Figure 10: Computed tomography sagittal section showing obvious gap at the fracture site. 
functional demand. There results concluded that patients who had patellar traction followed by tension band wiring had the best results in terms of functional outcome [2]. A study by Uvaraj., et al. has compared tension band wiring to patellectomies in neglected patella fractures. They reported reduction of the efficiency of the extensor mechanism by about $30 \%$ in patients after patellectomies. Hence, they concluded that TBW is superior to patellectomies in terms of functional outcome [3]. Surgical treatment is fraught with many complications mobilizing the quadriceps with fractional lengthening, $\mathrm{V}-\mathrm{Y} / \mathrm{Z}$ plasty requires a lot of soft tissue release and scarring thereby causing more stiffness, increased risk of infection, devitalizing the blood supply to patella. Postoperative management in these cases also requires a long immobilization period for healing thus making the results less favourable. Partial or total patellectomy may be required when the patella is not salvageable. Klassen and Trousdale [4] reported need for partial or total patellectomy when the fracture pattern or fragment size made internal fixation impossible. No specific recommendation regarding the use of bone grafts exists in literature. Patella non-union is disabling because of poor quadriceps function and functional outcome. The biggest challenge in treatment of patella non-union is difficulty in approximating displaced fragments due to soft tissue contractures. Preserved quadriceps function with complete knee range of motion in the scenario of patella non-union is a rare entity [5].

\section{Conclusion}

Patella forms an integral part of extensor mechanism of knee. Stable construct to fix the patella fracture is of prime importance for normal knee flexion and extension. Non-union and delayed union of fractures of patella are very uncommon. Our patient had a two-part transverse patella fracture for which ORIF with TBW and cerclage was done. The fracture was mal-reduced being evident on immediate post-operative radiograph. Later on, patient underwent debridement and implant removal in context of infection and patella non-union eventually healed by pseudoarthrosis. Despite of patella non-union, patient had complete knee range of motion and normal gait with no functional disability.

This brings up the question whether we really need to treat non-union of patella in low demand patient such as our patient.

\section{Acknowledgement}

The authors would like to express gratitude towards the patient and his family for allowing us to use the information for medical documentation and research purpose that led to the present article. There has been no contribution from any other person apart from the three mentioned authors.

\section{Bibliography}

1. Gwinner C., et al. "Current concepts review; Fractures of the patella”. GMS Interdisciplinary Plastic and Reconstructive Surgery DGPW 5 (2016).

2. Garg P., et al. "Patellar non-unions ; Comparison of various surgical methods of treatment". Indian Journal of Orthopaedics 46 (2012): 304-11.

3. Uvaraj NR., et al. "Surgical management of neglected fractures of the patella". Injury 38.8 (2007): 979-983.

4. Klassen JF and Trousdale RT. "Treatment of delayed and nonunion of the patella". Journal of Orthopaedic Trauma 11.3 (1997): 188-194.

5. Supreeth Nekkanti., et al. "An Unusual Presentation of Nonunion of patella with full range of movements of the knee joint". Journal of Integrative Oncology 5 (2016): 2.

\section{Assets from publication with us}

- Prompt Acknowledgement after receiving the article

- Thorough Double blinded peer review

- Rapid Publication

- Issue of Publication Certificate

- High visibility of your Published work

Website: www.actascientific.com/

Submit Article: www.actascientific.com/submission.php Email us: editor@actascientific.com

Contact us: +919182824667 